\title{
Crystal structure of 4-benzoyl-3-methyl-1-phenyl-2-pyrazolin-5-one sulfadiazine dimethylformamide monosolvate, $\mathrm{C}_{30} \mathrm{H}_{29} \mathrm{~N}_{7} \mathrm{O}_{4} \mathrm{~S}$
}

\author{
Omoruyi G. Idemudia ${ }^{*}$, , Alexander P. Sadimenko ${ }^{\mathrm{I}}$ and Eric C. Hosten ${ }^{\mathrm{II}}$ \\ ${ }^{\text {I }}$ Department of Chemistry, University of Fort Hare, Private Bag X1314, Alice 5700, South Africa \\ ${ }^{\text {II }}$ Department of Chemistry, Nelson Mandela Metropolitan University, PO Box 77000, Port Elizabeth 6031, South Africa
}

Received June 26, 2014, accepted October 20, 2014, available online October 24, 2014, CCDC no. 1267/4211

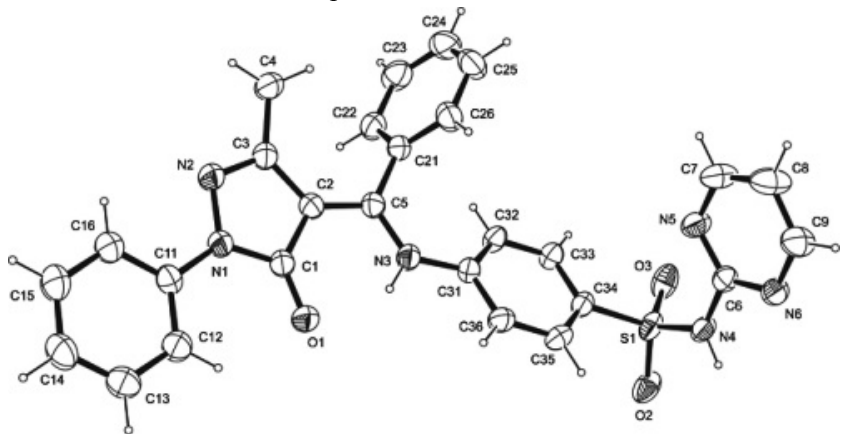

\section{Abstract}

$\mathrm{C}_{30} \mathrm{H}_{29} \mathrm{~N}_{7} \mathrm{O}_{4} \mathrm{~S}$, triclinic, $P \overline{1}$ (no. 2), $a=7.7793(3) \AA$, $b=9.1053(4) \AA, c=21.7108(9) \AA, \alpha=97.433(2)^{\circ}$,

$\beta=93.025(2)^{\circ}, \gamma=111.055(2)^{\circ}, V=1414.9 \AA^{3}, Z=2$, $R_{\mathrm{gt}}(F)=0.0399, w R_{\mathrm{ref}}\left(F^{2}\right)=0.1084, T=200 \mathrm{~K}$.

Table 1. Data collection and handling.

$\begin{array}{ll}\text { Crystal: } & \text { yellow platelets, } \\ & \text { size } 0.086 \times 0.360 \times 0.507 \mathrm{~mm} \\ \text { Wavelength: } & \text { Mo } K_{\alpha} \text { radiation }(0.71073 \AA) \\ \mu: & 1.64 \mathrm{~cm}^{-1} \\ \text { Diffractometer, scan mode: } & \text { Bruker APEX-II CCD, } \varphi \text { and } \omega \\ 2 \theta_{\text {max }}: & 56.74^{\circ} \\ N(h k l)_{\text {measured }}, N(h k l)_{\text {unique }}: & 38010,7042 \\ \text { Criterion for } I_{\text {obs }}, N(h k l)_{\text {gt }}: & I_{\text {obs }}>2 \sigma\left(I_{\text {obs }}\right), 6091 \\ N(\text { param })_{\text {refined }}: & 426 \\ \text { Programs: } & \text { SHELXS, SAINT, SADABS, SHELXLE, } \\ & \text { ORTEP-3; PLATON, MERCURY [11-16] }\end{array}$

\section{Source of material}

The reagents were of analytical grade and were used as obtained. To a solution of synthesized 4-benzoyl-3-methyl-1-phenyl-2pyrazolin-5-one $(2.0 \mathrm{mmol}, 0.56 \mathrm{~g})$ in methanol $(10 \mathrm{~mL})$ was added slowly to sulfadiazine $(2.0 \mathrm{mmol}, 0.50 \mathrm{~g})$ in methanol (10 $\mathrm{mL}$ ) while stirring, and the mixture was refluxed for $3 \mathrm{~h}$. The pale yellow precipitate obtained was filtered, washed with methanol, dried at room temperature and stored over fused $\mathrm{CaCl}_{2}$. Yellow cubic single crystals of the titled ketoimine Schiff base good enough for x-ray diffraction were grown at room temperature from slow evaporation of dimethylformamide solution.

\section{Experimental details}

Carbon-bound $\mathrm{H}$ atoms were placed in calculated positions and were included in the refinement in the riding model approximation, with $U_{\text {iso }}(\mathrm{H})$ set to $1.2 U_{\text {eq }}(\mathrm{C})$. The $\mathrm{H}$ atoms of the methyl groups were allowed to rotate with a fixed angle around the $\mathrm{C}-\mathrm{C}$ bond to best fit the experimental electron density (HFIX 137 in the SHELX program suite [11]), with $U_{\text {iso }}(\mathrm{H})$ set to $1.5 U_{\text {eq }}(\mathrm{C})$.
The nitrogen-bound $\mathrm{H}$ atoms were located on a difference Fourier map and refined freely. The solvent dimethylformamide is positionally disordered and various anisotropic displacement parameters and bond lengths for the solvent were restrained.

\section{Discussion}

The chemistry of acylpyrazolone derived heterocycles has received a lot of research attention because of their interesting reactivity and useful applications [1]. Their strong chelating property, the reason they are excellent analytical reagents, have been improved by way of forming superior group of coordination compounds known as Schiff bases that are bioactive [2, 3]. The therapeutic significant of sulfa drugs derivatives have been well established $[4,5]$. The amine $-\mathrm{NH}_{2}$ group they possess is an excellent nucleophile target site for electrophilic carbonyl carbon in aldehydes and ketones to form Schiff bases [6, 7]. We have reported the crystal structure of 4-benzoyl-3- methyl-1-phenyl-2pyrazolin-5-one Schiff bases with phenylhydrazine derivatives $[3,8]$. In a probe into the possible synergistic effect attributed to the combination of sulfadiazine and 4-acylpyrazolone, presented herein is the crystal and molecular structure of a 4-benzoyl-3methyl-1-phenyl-2-pyrazolin-5-one Schiff base with sulfadiazine. In the asymmetric unit the title compound occurs together with one dimethylformamide solvent molecule. There are two notable intramolecular hydrogen bonds, both with $\mathrm{O} 1$, namely $\mathrm{N} 3-\mathrm{H} 3 \cdots \mathrm{O} 1$ and $\mathrm{C} 12-\mathrm{H} 12 \cdots \mathrm{O} 1$ that hold the phenylpyrazolone backbone essentially planar together with extended resonance. The least square plane through the atoms N1$\mathrm{N} 3, \mathrm{O} 1, \mathrm{C} 1-\mathrm{C} 3, \mathrm{C} 5$, and C11-C16 has a r.m.s. of $0.0671 \AA$. Adjacent molecules are held together at the sulfadiazine group with two short N4-H4 N.N6 hydrogen bonds with lengths of 2.062(19) $\AA$ which in terms of graph-set analyses $[9,10]$ can be described by means of a $R_{2}^{2}(8)$ descriptor on the unary level. There are also a large number of $\pi \cdots \pi$ ring interactions. The two shortest interactions occur with the phenyl ring C31-C36 which has two $\pi \cdots \pi$ ring interactions; one with an adjacent pyrazole ring N1, N2, C1$\mathrm{C} 3$, and one with the phenyl ring $\mathrm{C} 11-\mathrm{C} 16$. The centroid to centroid distances are 4.2512(8) and 4.2530(9) $\AA$, respectively. The crystal contains the solvent dimethylformamide positionally disordered in a 0.82:0.18 ratio.

\footnotetext{
* Correspondence author (e-mail: oidemudia@ufh.ac.za)
} 
Table 2. Atomic coordinates and displacement parameters (in $\AA^{2}$ ).

\begin{tabular}{llllll}
\hline Atom & Site Occ. & $x$ & $y$ & $z$ & $U_{\text {iso }}$ \\
\hline $\mathrm{H}(3)$ & $2 i$ & $0.047(2)$ & $0.942(2)$ & $0.2669(8)$ & $0.048(5)$ \\
$\mathrm{H}(4)$ & $2 i$ & $0.562(2)$ & $0.610(2)$ & $0.0528(9)$ & $0.041(4)$ \\
$\mathrm{H}(4 \mathrm{~A})$ & $2 i$ & -0.2662 & 0.6735 & 0.4337 & 0.074 \\
$\mathrm{H}(4 \mathrm{~B})$ & $2 i$ & -0.3631 & 0.7728 & 0.4756 & 0.074 \\
$\mathrm{H}(4 \mathrm{C})$ & $2 i$ & -0.1439 & 0.8186 & 0.4867 & 0.074 \\
$\mathrm{H}(7)$ & $2 i$ & 0.2081 & 0.2030 & 0.1803 & 0.058 \\
$\mathrm{H}(8)$ & $2 i$ & 0.0906 & 0.0282 & 0.0874 & 0.066 \\
$\mathrm{H}(9)$ & $2 i$ & 0.1914 & 0.1181 & -0.0049 & 0.056 \\
$\mathrm{H}(12)$ & $2 i$ & -0.2714 & 1.2294 & 0.2686 & 0.050 \\
$\mathrm{H}(13)$ & $2 i$ & -0.4423 & 1.3869 & 0.2505 & 0.057 \\
$\mathrm{H}(14)$ & $2 i$ & -0.6622 & 1.4072 & 0.3156 & 0.061 \\
$\mathrm{H}(15)$ & $2 i$ & -0.7155 & 1.2655 & 0.3990 & 0.063 \\
$\mathrm{H}(16)$ & $2 i$ & -0.5421 & 1.1116 & 0.4196 & 0.051 \\
$\mathrm{H}(22)$ & $2 i$ & 0.1920 & 0.8998 & 0.4533 & 0.046 \\
$\mathrm{H}(23)$ & $2 i$ & 0.2664 & 0.7236 & 0.5088 & 0.058 \\
$\mathrm{H}(24)$ & $2 i$ & 0.1791 & 0.4578 & 0.4647 & 0.063 \\
$\mathrm{H}(25)$ & $2 i$ & 0.0193 & 0.3653 & 0.3656 & 0.058 \\
$\mathrm{H}(26)$ & $2 i$ & -0.0516 & 0.5411 & 0.3084 & 0.046 \\
\hline
\end{tabular}

Table 2. continued.

\begin{tabular}{|c|c|c|c|c|c|c|}
\hline Atom & Site & Occ. & $x$ & $y$ & $z$ & $U_{\text {iso }}$ \\
\hline $\mathrm{H}(32)$ & $2 i$ & & 0.3556 & 0.7837 & 0.3296 & 0.039 \\
\hline $\mathrm{H}(33)$ & $2 i$ & & 0.5666 & 0.7108 & 0.2723 & 0.039 \\
\hline $\mathrm{H}(35)$ & $2 i$ & & 0.3543 & 0.8095 & 0.1153 & 0.042 \\
\hline $\mathrm{H}(36)$ & $2 i$ & & 0.1343 & 0.8707 & 0.1715 & 0.041 \\
\hline H(71_a) & $2 i$ & 0.821 & 0.6264 & 0.0735 & 0.0489 & 0.108 \\
\hline $\mathrm{H}\left(72 \overline{\mathrm{A}} \_\mathrm{a}\right)$ & $2 i$ & 0.821 & 0.9685 & 0.5046 & 0.1175 & 0.137 \\
\hline $\mathrm{H}\left(72 \mathrm{~B} \_\mathrm{a}\right)$ & $2 i$ & 0.821 & 0.8766 & 0.4823 & 0.0475 & 0.137 \\
\hline $\mathrm{H}\left(72 \mathrm{C}^{-} \mathrm{a}\right)$ & & 0.821 & 1.0400 & 0.4191 & 0.0609 & 0.137 \\
\hline H(73A_a) & $2 i$ & 0.821 & 0.8841 & 0.2614 & 0.1705 & 0.115 \\
\hline $\mathrm{H}\left(73 \mathrm{~B} \_\mathrm{a}\right)$ & $2 i$ & 0.821 & 0.6728 & 0.1497 & 0.1479 & 0.115 \\
\hline $\mathrm{H}\left(73 \mathrm{C}_{-}^{-} \mathrm{a}\right)$ & $2 i$ & 0.821 & 0.7275 & 0.3369 & 0.1699 & 0.115 \\
\hline $\mathrm{H}\left(81 \_\bar{b}\right)$ & $2 i$ & 0.179 & 0.9766 & 0.4269 & 0.0437 & 0.108 \\
\hline $\mathrm{H}\left(82 \overline{\mathrm{A}} \_\mathrm{b}\right)$ & $2 i$ & 0.179 & 1.0490 & 0.4666 & 0.1460 & 0.138 \\
\hline $\mathrm{H}\left(82 \mathrm{~B} \_\mathrm{b}\right)$ & $2 i$ & 0.179 & 0.9491 & 0.3261 & 0.1843 & 0.138 \\
\hline $\mathrm{H}\left(82 \mathrm{C}^{-} \mathrm{b}\right)$ & $2 i$ & 0.179 & 0.8586 & 0.4545 & 0.1737 & 0.138 \\
\hline $\mathrm{H}\left(83 \mathrm{~A} \_\mathrm{b}\right)$ & $2 i$ & 0.179 & 0.7227 & 0.0885 & 0.1298 & 0.101 \\
\hline $\mathrm{H}\left(83 \mathrm{~B} \_\mathrm{b}\right)$ & $2 i$ & 0.179 & 0.6013 & 0.1005 & 0.0701 & 0.101 \\
\hline $\mathrm{H}\left(83 \mathrm{C}^{-} \mathrm{b}\right)$ & $2 i$ & 0.179 & 0.5974 & 0.1945 & 0.1373 & 0.101 \\
\hline
\end{tabular}

Table 3. Atomic coordinates and displacement parameters (in $\AA^{2}$ ).

\begin{tabular}{|c|c|c|c|c|c|c|c|c|c|c|c|}
\hline Atom & Site & Occ. & $x$ & $y$ & $z$ & $U_{11}$ & $U_{22}$ & $U_{33}$ & $U_{12}$ & $U_{13}$ & $U_{23}$ \\
\hline $\mathrm{S}(1)$ & $2 i$ & & $0.65134(4)$ & $0.71256(4)$ & $0.14880(2)$ & $0.0290(2)$ & $0.0400(2)$ & $0.0296(2)$ & $0.0072(1)$ & $0.0086(1)$ & $-0.0018(1)$ \\
\hline $\mathrm{O}(1)$ & $2 i$ & & $-0.0871(1)$ & $1.0861(1)$ & $0.27987(5)$ & $0.0474(6)$ & $0.0444(5)$ & $0.0352(5)$ & $0.0257(5)$ & $0.0126(4)$ & $0.0148(4)$ \\
\hline $\mathrm{O}(2)$ & $2 i$ & & $0.7569(2)$ & $0.8474(1)$ & $0.12137(5)$ & $0.0421(6)$ & $0.0431(6)$ & $0.0415(6)$ & $-0.0034(5)$ & $0.0184(5)$ & $-0.0031(5)$ \\
\hline $\mathrm{O}(3)$ & $2 i$ & & $0.7472(1)$ & $0.6494(2)$ & $0.18952(5)$ & $0.0348(5)$ & $0.0673(7)$ & $0.0391(6)$ & $0.0242(5)$ & $0.0042(4)$ & $-0.0014(5)$ \\
\hline $\mathrm{N}(1)$ & $2 i$ & & $-0.2821(2)$ & $1.0651(1)$ & $0.35996(5)$ & $0.0392(6)$ & $0.0357(6)$ & $0.0303(5)$ & $0.0206(5)$ & $0.0070(4)$ & $0.0078(4)$ \\
\hline $\mathrm{N}(2)$ & $2 i$ & & $-0.3276(2)$ & $0.9769(1)$ & $0.40889(5)$ & $0.0447(6)$ & $0.0415(6)$ & $0.0318(6)$ & $0.0244(5)$ & $0.0104(5)$ & $0.0112(5)$ \\
\hline $\mathrm{N}(4)$ & $2 i$ & & $0.5405(2)$ & $0.5793(1)$ & $0.08783(5)$ & $0.0394(6)$ & $0.0329(6)$ & $0.0258(5)$ & $0.0062(5)$ & $0.0106(5)$ & $0.0017(4)$ \\
\hline $\mathrm{N}(5)$ & $2 i$ & & $0.3696(2)$ & $0.3865(2)$ & $0.14430(6)$ & $0.0437(6)$ & $0.0413(6)$ & $0.0311(6)$ & $0.0118(5)$ & $0.0072(5)$ & $0.0100(5)$ \\
\hline $\mathrm{N}(6)$ & $2 i$ & & $0.3584(2)$ & $0.3353(1)$ & $0.03312(5)$ & $0.0362(6)$ & $0.0329(6)$ & $0.0316(6)$ & $0.0080(5)$ & $0.0049(4)$ & $0.0037(4)$ \\
\hline $\mathrm{C}(1)$ & $2 i$ & & $-0.1501(2)$ & $1.0310(2)$ & $0.32707(6)$ & $0.0364(6)$ & $0.0319(6)$ & $0.0296(6)$ & $0.0167(5)$ & $0.0047(5)$ & $0.0044(5)$ \\
\hline $\mathrm{C}(2)$ & $2 i$ & & $-0.1049(2)$ & $0.9187(2)$ & $0.35976(6)$ & $0.0385(7)$ & $0.0320(6)$ & $0.0293(6)$ & $0.0174(5)$ & $0.0056(5)$ & $0.0064(5)$ \\
\hline$C(3)$ & $2 i$ & & $-0.2242(2)$ & $0.8917(2)$ & $0.40871(6)$ & $0.0423(7)$ & $0.0383(7)$ & $0.0321(6)$ & $0.0224(6)$ & $0.0091(5)$ & $0.0082(5)$ \\
\hline $\mathrm{C}(4)$ & $2 i$ & & $-0.2517(3)$ & $0.7795(2)$ & $0.45520(8)$ & $0.060(1)$ & $0.061(1)$ & $0.0494(9)$ & $0.0390(8)$ & $0.0272(8)$ & $0.0290(8)$ \\
\hline$C(5)$ & $2 i$ & & $0.0191(2)$ & $0.8488(2)$ & $0.33967(6)$ & $0.0347(6)$ & $0.0287(6)$ & $0.0279(6)$ & $0.0134(5)$ & $0.0038(5)$ & $0.0028(5)$ \\
\hline$C(7)$ & $2 i$ & & $0.2474(2)$ & $0.2375(2)$ & $0.14221(8)$ & $0.0508(9)$ & $0.0496(9)$ & $0.0411(8)$ & $0.0091(7)$ & $0.0062(7)$ & $0.0222(7)$ \\
\hline $\mathrm{C}(8)$ & $2 i$ & & $0.1770(3)$ & $0.1333(2)$ & $0.08781(8)$ & $0.055(1)$ & $0.0421(8)$ & $0.052(1)$ & $-0.0031(7)$ & $-0.0033(8)$ & $0.0188(7)$ \\
\hline $\mathrm{C}(9)$ & $2 i$ & & $0.2375(2)$ & $0.1881(2)$ & $0.03370(8)$ & $0.0511(9)$ & $0.0367(7)$ & $0.0409(8)$ & $0.0036(6)$ & $-0.0032(7)$ & $0.0044(6)$ \\
\hline $\mathrm{C}(11)$ & $2 i$ & & $-0.3873(2)$ & $1.1582(2)$ & $0.34669(6)$ & $0.0352(6)$ & $0.0323(6)$ & $0.0326(6)$ & $0.0164(5)$ & $-0.0001(5)$ & $0.0022(5)$ \\
\hline $\mathrm{C}(12)$ & $2 i$ & & $-0.3601(2)$ & $1.2380(2)$ & $0.29594(7)$ & $0.0482(8)$ & $0.0511(8)$ & $0.0366(7)$ & $0.0283(7)$ & $0.0068(6)$ & $0.0114(6)$ \\
\hline$C(13)$ & $2 i$ & & $-0.4629(2)$ & $1.3307(2)$ & $0.28506(8)$ & $0.0536(9)$ & $0.0557(9)$ & $0.0434(8)$ & $0.0306(8)$ & $0.0025(7)$ & $0.0163(7)$ \\
\hline $\mathrm{C}(14)$ & $2 i$ & & $-0.5933(2)$ & $1.3426(2)$ & $0.32322(8)$ & $0.0545(9)$ & $0.057(1)$ & $0.057(1)$ & $0.0375(8)$ & $0.0046(8)$ & $0.0140(8)$ \\
\hline$C(15)$ & $2 i$ & & $-0.6234(2)$ & $1.2597(2)$ & $0.37279(9)$ & $0.0537(9)$ & $0.063(1)$ & $0.059(1)$ & $0.0398(9)$ & $0.0164(8)$ & $0.0171(8)$ \\
\hline$C(16)$ & $2 i$ & & $-0.5209(2)$ & $1.1678(2)$ & $0.38504(7)$ & $0.0458(8)$ & $0.0476(8)$ & $0.0440(8)$ & $0.0264(7)$ & $0.0105(6)$ & $0.0130(7)$ \\
\hline $\mathrm{C}(21)$ & $2 i$ & & $0.0645(2)$ & $0.7370(2)$ & $0.37544(6)$ & $0.0329(6)$ & $0.0334(6)$ & $0.0334(6)$ & $0.0172(5)$ & $0.0089(5)$ & $0.0084(5)$ \\
\hline $\mathrm{C}(22)$ & $2 i$ & & $0.1579(2)$ & $0.7913(2)$ & $0.43517(7)$ & $0.0450(8)$ & $0.0427(7)$ & $0.0321(7)$ & $0.0211(6)$ & $0.0073(6)$ & $0.0065(6)$ \\
\hline$C(23)$ & $2 i$ & & $0.2012(2)$ & $0.6868(2)$ & $0.46820(8)$ & $0.0521(9)$ & $0.067(1)$ & $0.0384(8)$ & $0.0329(8)$ & $0.0081(7)$ & $0.0181(7)$ \\
\hline $\mathrm{C}(24)$ & $2 i$ & & $0.1494(2)$ & $0.5295(2)$ & $0.44193(9)$ & $0.056(1)$ & $0.059(1)$ & $0.062(1)$ & $0.0366(8)$ & $0.0179(8)$ & $0.0303(9)$ \\
\hline$C(25)$ & $2 i$ & & $0.0552(2)$ & $0.4745(2)$ & $0.38310(9)$ & $0.0491(9)$ & $0.0348(7)$ & $0.070(1)$ & $0.0219(7)$ & $0.0148(8)$ & $0.0144(7)$ \\
\hline$C(26)$ & $2 i$ & & $0.0126(2)$ & $0.5785(2)$ & $0.34922(7)$ & $0.0379(7)$ & $0.0338(7)$ & $0.0462(8)$ & $0.0159(6)$ & $0.0064(6)$ & $0.0048(6)$ \\
\hline $\mathrm{C}(31)$ & $2 i$ & & $0.2240(2)$ & $0.8334(2)$ & $0.25587(6)$ & $0.0322(6)$ & $0.0282(6)$ & $0.0277(6)$ & $0.0120(5)$ & $0.0062(5)$ & $0.0027(5)$ \\
\hline$C(34)$ & $2 i$ & & $0.4803(2)$ & $0.7550(2)$ & $0.18908(6)$ & $0.0293(6)$ & $0.0299(6)$ & $0.0266(6)$ & $0.0077(5)$ & $0.0072(5)$ & $0.0007(5)$ \\
\hline$C(35)$ & $2 i$ & & $0.3529(2)$ & $0.8027(2)$ & $0.15859(6)$ & $0.0397(7)$ & $0.0416(7)$ & $0.0253(6)$ & $0.0137(6)$ & $0.0081(5)$ & $0.0090(5)$ \\
\hline $\mathrm{C}(36)$ & $2 i$ & & $0.2239(2)$ & $0.8401(2)$ & $0.19212(6)$ & $0.0380(7)$ & $0.0382(7)$ & $0.0303(6)$ & $0.0171(6)$ & $0.0061(5)$ & $0.0111(5)$ \\
\hline $\mathrm{N}\left(7 \_\mathrm{a}\right)$ & $2 i$ & 0.821 & $0.7975(5)$ & $0.2830(5)$ & $0.0847(1)$ & $0.066(2)$ & $0.066(1)$ & $0.039(2)$ & $0.037(1)$ & $0.011(1)$ & $0.012(1)$ \\
\hline $\mathrm{O}\left(7 \_\mathrm{a}\right)$ & $2 i$ & 0.821 & $0.7234(8)$ & $0.1756(6)$ & $-0.0149(1)$ & $0.264(6)$ & $0.158(4)$ & $0.038(1)$ & $0.128(4)$ & $-0.009(2)$ & $0.002(2)$ \\
\hline C(71_a) & $2 i$ & 0.821 & $0.7098(6)$ & $0.1701(4)$ & $0.0385(2)$ & $0.125(3)$ & $0.094(2)$ & $0.064(2)$ & $0.063(2)$ & $-0.011(2)$ & $0.002(2)$ \\
\hline C(72_a) & $2 i$ & 0.821 & $0.9312(5)$ & $0.4342(4)$ & $0.0770(2)$ & $0.093(2)$ & $0.077(2)$ & $0.123(3)$ & $0.042(2)$ & $0.060(2)$ & $0.032(2)$ \\
\hline $\mathrm{C}\left(73 \_a\right)$ & $2 i$ & 0.821 & $0.7681(5)$ & $0.2555(4)$ & $0.1484(1)$ & $0.105(2)$ & $0.104(2)$ & $0.050(2)$ & $0.066(2)$ & $0.029(2)$ & $0.024(2)$ \\
\hline $\mathrm{N}\left(8 \_\bar{b}\right)$ & $2 i$ & 0.179 & $0.827(3)$ & $0.294(2)$ & $0.0979(7)$ & $0.066(2)$ & $0.066(1)$ & $0.039(2)$ & $0.037(1)$ & $0.011(1)$ & $0.012(1)$ \\
\hline $\mathrm{O}\left(8 \_b\right)$ & $2 i$ & 0.179 & $0.812(3)$ & $0.257(3)$ & $-0.0079(9)$ & $0.14(1)$ & $0.15(2)$ & $0.12(1)$ & $0.01(1)$ & $-0.05(1)$ & $0.00(1)$ \\
\hline $\mathrm{C}\left(8 \overline{1} \_\mathrm{b}\right)$ & $2 i$ & 0.179 & $0.875(3)$ & $0.329(2)$ & $0.0427(6)$ & $0.125(3)$ & $0.094(2)$ & $0.064(2)$ & $0.063(2)$ & $-0.011(2)$ & $0.002(2)$ \\
\hline $\mathrm{C}\left(82 \_\mathrm{b}\right)$ & $2 i$ & 0.179 & $0.929(2)$ & $0.393(2)$ & $0.1551(7)$ & $0.09(1)$ & $0.074(9)$ & $0.10(1)$ & $0.021(8)$ & $0.023(8)$ & $-0.017(8)$ \\
\hline $\mathrm{C}\left(83 \_\mathrm{b}\right)$ & $2 i$ & 0.179 & $0.675(2)$ & $0.159(2)$ & $0.1097(6)$ & $0.079(8)$ & $0.070(7)$ & $0.065(8)$ & $0.040(6)$ & $0.015(6)$ & $0.015(6)$ \\
\hline
\end{tabular}


Acknowledgments. The financial support of the Govan Mbeki Research and Development Centre (GMRDC), University of Fort Hare and National Research Foundation (NRF) is appreciated. OGI thank the NRF for the award of a PDF (Grant UID: 92275).

\section{References}

1. Marchetti, F.; Pettinari, C.; Pettinari, R.: Acylpyrazolone ligands: Synthesis, structures, metal coordination chemistry and applications. Coord. Chem. Rev. 249 (2005) 2909-2945.

2. Yang, Z. Y.; Yang, R. D.; Li, F. S.; Yu, K. B.: Crystal structure and antitumor activity of some rare earth metal complexes with Schiff base. Polyhedron 19 (2000) 2599-2604.

3. Idemudia, O. G., Sadimenko, A. P., Afolayan, A. J.; Hosten, E. C.: 3Methyl-1-phenyl-4-[(phenyl)(2-phenylhydrazin-1-yl)methylidene]- $1 H$ pyrazol-5(4H)-one. Acta Crystallogr. E68 (2012) o1280-o1281.

4. Owa, T.; Nagasu, T.: Novel Sulfonamide derivatives for the treatment of cancer. Exp. Opin. Ther. Pat. 10 (2000) 1725-1740.

5. Fathalla, O. A.; Awad S. M.; Mohamed M. S.: Synthesis of new 2- thiouracil-5-sulphonamide derivatives with antibacterial and antifungal activity. Arch. Pharm. Res. 28 (2005) 1205-1211.

6. Valarmathy, G.; Subbalakshmi, R.: Synthesis, spectra characterization of biologically active novel schiff base complexes derived from 2sulfanilamidopyrimidine. Int J Pharm Bio Sci. 4 (2013) 1019-1029.

7. Vellaiswamy, G.; Ramaswamy, S.: Synthesis, spectra characterization and antimicrobial screening of novel schiff bases from sulfa drugs. Int J Pharm. Pharm. Sci. 6 (2014) 487-491.
8. Idemudia, O. G.; Sadimenko, A. P.; Hosten, E. C.: 4-\{[2-(2,4Dinitrophenyl)hydrazinylidene](phenyl)methyl $\}$-5-methyl-2-phenyl$1 H$-pyrazol-3(2H)-one ethanol monosolvate. Acta Crystallogr. E68 (2012) o3380.

9. Bernstein, J.; Davis, R. E.; Shimoni, L.; Chang, N.-L.: Patterns in Hydrogen Bonding: Functionality and Graph Set Analysis in Crystals. Angew. Chem. Int. Ed. Engl. 34 (1995) 1555-1573.

10. Etter, M. C.; MacDonald, J. C.; Bernstein, J.: Graph-set analysis of hydrogen-bond patterns in organic crystals. Acta Crystallogr. B46 (1990) 256-262.

11. Sheldrick, G. M.: A short history of SHELX. Acta Crystallogr. A64 (2008) 112-122.

12. Bruker APEXII, SAINT, SADABS. Bruker AXS Inc., Madison, Wisconsin, USA (2007).

13. Hübschle, C. B.; Sheldrick, G. M.; Dittrich, B.: ShelXle: a Qt graphical user interface for SHELXL. J. Appl. Cryst. 44 (2011) 1281-1284.

14. Farrugia, L. J.: WinGX and ORTEP for Windows: an update. J. Appl. Crystallogr. 45 (2012) 849-854.

15. Spek, A. L.: Structure validation in chemical crystallography. Acta Crystallogr. D65 (2009) 148-155.

16. Macrae, C. F.; Bruno, I. J.; Chisholm, J. A.; Edgington, P. R.; McCabe, P.; Pidcock, E.; Rodriguez-Monge, L.; Taylor, R.; van de Streek, J.; Wood, P.A.: Mercury CSD 2.0 - new features for the visualization and investigation of crystal structures. J. Appl. Crystallogr. 41 (2008) 466-470. 